\title{
IMAGING OF ROTATOR CUFF PATHOLOGY- IS ULTRASOUND AS GOOD AS MRI
}

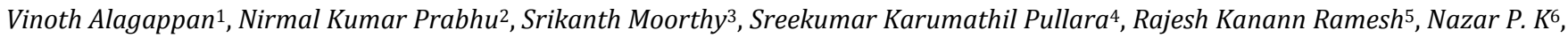
Chinmay Kulkarni ${ }^{7}$, Rajsekar Chandrasekharan ${ }^{8}$

${ }_{1}^{1}$ Senior Resident, Department of Radiology, Amrita Institute of Medical Sciences and Research Institute, Kerala.

${ }^{2}$ Professor, Department of Radiology, Amrita Institute of Medical Sciences and Research Institute, Kerala.

3 Professor, Department of Radiology, Amrita Institute of Medical Sciences and Research Institute, Kerala.

${ }^{4}$ Professor, Department of Radiology, Amrita Institute of Medical Sciences and Research Institute, Kerala.

${ }_{5}^{5}$ Associate Professor, Department of Radiology, Amrita Institute of Medical Sciences and Research Institute, Kerala.

${ }^{6}$ Associate Professor, Department of Radiology, Amrita Institute of Medical Sciences and Research Institute, Kerala.

${ }^{7}$ Assistant Professor, Department of Radiology, Amrita Institute of Medical Sciences and Research Institute, Kerala.

${ }^{8}$ Assistant Professor, Department of Radiology, Amrita Institute of Medical Sciences and Research Institute, Kerala.

\section{ABSTRACT}

Aim of this study is to compare the diagnostic accuracy of ultrasound versus MRI in detecting rotator cuff pathology. Rotator cuff pathology involves group of disorder, which progresses from bursitis to tendinitis to partial tear and full thickenss tear. The most commonly affected tendon is the supraspinatus tendon. In our prospective study of 35 patients as initial ultrasound was followed by MRI sensitivity, specificity and accuracy for diagnosis of full thickness tear of supraspinatus when USG was compared to MRI was $100 \%$, whereas sensitivity, specificity and accuracy of diagnosing partial thickness tear using ultrasound compared to MRI are $100 \%, 78 \%$ and $84 \%$. There is no significant $p$ value difference on comparing ultrasound with MRI on detecting tears. Ultrasound and MRI showed almost similar diagnostic accuracy in associated findings like tendon retraction, muscle atrophy, bursal effusion. Ultrasound also has additional value of dynamic evaluation, which is useful in evaluating subacromial and subcoracoid impingement. Modality choice for the evaluation of rotator cuff pathology should be based on several factors like availability, patient preference and clinical information being sought. The high resolution sonography is an attractive screening modality for rotator cuff in patients presenting with painful shoulder. A well performed ultrasound examination in most cases obviates the need for more invasive diagnostic tests like arthrography and cumbersome and expensive MRI examinations.

\section{KEYWORDS}

Rotator Cuff, Magnetic Resonance Imaging, Ultrasound, Shoulder.

HOW TO CITE THIS ARTICLE: Vinoth Alagappan, Nirmal Kumar Prabhu, Srikanth Moorthy, Sreekumar Karumathil Pullara, Rajesh Kanann Ramesh, Nazar P. K, Chinmay Kulkarni, Rajsekar Chandrasekharan. "Imaging of Rotator Cuff Pathology - Is Ultrasound as Good as MRI." Journal of Evolution of Medical and Dental Sciences 2015; Vol. 4, Issue 100, December 14; Page: 16567-16572, DOI: $10.14260 /$ jemds/2015/2467

\section{INTRODUCTION}

The shoulder joint is an incongruous ball and socket joint without any fixed axis of rotation, which has wider range of motion in multiple planes, hence stability is compromised for mobility. To compensate for this unstable bone anatomy the shoulder is protected anteriorly, posteriorly and superiorly by a capsule and rotator cuff tendons.

These tendons are subjected to wear and tear during day-to-day activities giving rise to shoulder pain. The rotator cuff muscles are namely supraspinatus, infraspinatus, subscapularis and teres minor. The common disorders involving the rotator cuff tendons include impingement, tendinopathy and tear.

It is a dynamic process leading on from degeneration to tears in these tendons. The most commonly affected rotator cuff tendon is the supraspinatus. The factors which aid on to this progress includes age, occupation, trauma, acromion type,

Financial or Other, Competing Interest: None.

Submission 23-11-2015, Peer Review 24-11-2015,

Acceptance 06-12-2015, Published 14-12-2015.

Corresponding Author:

Vinoth Alagappan,

Department of Radiology,

Amrita Institute of Medical Sciences and Research Institute.

Ponekkara, Cochin-682041,

Kerala.

E-mail: vinothsrmc@gmail.com

DOI:10.14260/jemds/2015/2467 slope and position, acromio-clavicular joint degeneration, proximal migration of the humeral head, bony spurs compressing on the tendons.

The tears are further classified as complete/full thickness and partial tears depending on the involvement of the whole or part of the tendon. Contrast arthrography has long been the premier radiological examination used to diagnose pathologies of the rotator cuff.(1) Unfortunately, arthrography is an invasive procedure relatively expensive and time-consuming.(2)

Two competing non-invasive imaging techniques, Ultrasound (USG) and Magnetic Resonance Imaging (MRI), are taking over the role of arthrography. Both MRI and USG are widely used for the evaluation of pathologic conditions of the rotator cuff and essentially obviate conventional arthrography. Ultrasound being relatively inexpensive and easily available, the purpose of this study is to evaluate the diagnostic accuracy of ultrasound in comparison to MRI on detecting rotator cuff pathologies.

\section{MATERIALS AND METHOD}

Study design: Prospective study.

Study setting:Cases referred to Radiology Department for MRI with suspision rotator cuff tear.

Study period: Oct 2012-Oct 2014

Sample size : 34 


\section{Inclusion Criteria}

- History of pain in the shoulder and restriction of movements.

- History of trauma.

- Clinically suspected to have rotator cuff pathology.

\section{Exclusion Criteria}

- Previous history of surgery or prosthesis.

- Patients with pacemakers, metallic implants in the body or with claustrophobia.

\section{Study Method}

All the patients underwent Ultrasound followed by MRI after giving an informed consent.

Ultrasound examination of the suspected shoulder including dynamic assessment was performed using Philips IU 22 Machine with L12-5 MHZ probe.

MRI examination of the affected shoulder was done using GE Medical Systems, Milwaukee, Wisconsin-1.5 Tesla HDXT using specific shoulder coil placed in the affected shoulder with patient in supine position. Multiplanar imaging were obtained in axial PDFS, Oblique coronal PDFS, oblique sagittal PDFS and Oblique coronal T1.

\section{Statistical Analysis}

SPSS statistical software. McNemar's Chi-Square's test was applied. If the $\mathrm{p}$ value is found to be $>0.05$, the disagreement between the two methods was found to be statistically not significant.

\section{RESULTS}

Rotator cuff pathologies were noted in 19 female patients and 16 male patients, out of which 26 were found in the age group of 50-70 and 9 below 50 years.

Both USG and MRI detected 34 patients with rotator cuff pathology involving supraspinatus tendon. Subscapularis pathology was noted in 6 patients using ultrasound and in 7 patients using MRI. Infraspinatus pathology was noted in 2 patients using ultrasound and 4 patients using MRI. One patient had subacromial subdeltoid bursitis in both ultrasound and MRI without any rotator cuff pathology.

USG evaluation of supraspinatus tendon showed 15 patients with full thickness tear, 7 patients with partial thickness tear, 2 patients with tendinopathy alone, 10 patients with both tendinopathy and tear, whereas MRI showed 15 patients with full thickness tear, 5 patients with partial thickness tear, 7 patients with tendinopathy, 7 patients with both tendinopathy and tear.

Both ultrasound and MRI showed 6 patients having subscapularis pathology - 2 with tear, 3 with tendinopathy and 1 with both tendinopathy and tear. Two patients had infraspinatus partial tear. All the tears of subscapularis and infraspinatus were associated with supraspinatus pathology.

Out of 32 supraspinatus tears on USG, 27 showed similar tears in MRI, 5 partial thickness tears diagnosed with associated tendinopathic changes using ultrasound showed only tendinopathic changes without any tear in MRI.

There are 4 patients (14\%) with full thickness tear of supraspinatus tendon associated with transverse humeral ligament tear and subluxation of biceps tendon and all were diagnosed in USG and MRI.
Error in tear width measurement in ultrasound compared to MRI is noted in 3 of 18 full thickness tears and 7 of 14 partial thickness tear.

Retraction of supraspinatus tendon were identified in $15(43 \%)$ patients, out of which ultrasound correlated with MRI in 12 patients.

There was a total of five patients with supraspinatus muscle atrophy and one with infraspinatus muscle atrophy. Two out of this 5 patients were identified using both ultrasound and MRI, whereas 3 were identified using only MRI. One infraspinatus muscle atophy was identified only in MRI and one was identified using both ultrasound and MRI None of them showed subscapularis or teres minor atrophy.

\section{Associated Changes}

Ultrasound showed 12 patients with normal AC joint, 22 with AC joint arthropathic changes associated with supraspinatus pathology and 1 with AC joint changes, but no supraspinatus pathology, whereas MRI showed 13 patients with normal AC joint, 19 with AC joint arthropathic changes associated with rotator cuff pathology, 1 with AC joint changes but no rotator cuff pathology and 2 with os acromiale.

Thirty four patients had subacromial subdeltoid bursa effusion with rotator cuff changes and one had subacromial subdeltoid bursa changes without any rotator cuff pathologies using ultrasound, and 33 patients with both subacromial subdeltoid bursa and rotator cuff changes, one with only subacromial subdeltoid bursal effusion and one with normal subacromial subdeltoid bursa in MRI. Bicepital groove effusion was noted in nine patients using USG and ten patients using MRI.

Twenty eight patients were identified with normal CAL (Coraco Acromial Ligament) and 7 with CAL thickening in ultrasound, whereas in MRI 25 were identified with normal CAL, 10 with CAL thickening.

The presence of enthesophytes with supraspinatus pathology were found in five patients using ultrasound compared to 14 patients using MRI.

Assessment of subacromial and subcoracoid impingement.

In dynamic assesment of shoulder for subacromial space using ultrasound results of 4 patients were inconclusive as they could not perform dynamic maneuvers due to extreme pain and restriction of movements. Twenty one patients had impingement and 10 were diagnosed as not having impingement, whereas MRI findings showed only 10 as having significant impingement.

Subcoracoid impingement study was inconclusive in five patients, impingement was present in four patients and absent in 26 patients. Ultrasound and MRI showed all the patients with impingement equally.

\section{DISCUSSION}

Rotator cuff pathologies are one of the most common causes of the shoulder pain and dysfunction in patients over 40 years of age. Tendon tear demonstrates a sequential progression from the partial thickness tear almost always starting in the supraspinatus to massive tears involving multiple cuff tendons. Tear is almost always peripheral near the attachment of the cuff to the tuberosities and always begins in the supraspinatus part of the rotator cuff near the biceps tendon. 
A full thickness tear involves tendon defect, which extends all the way from articular to the bursal surface of the rotator cuff and a partial thickness tear involves only the superficial surface, mid-substance or deep surface.

Various techniques were used for evaluating these rotator cuff pathologies after clinical evaluation like arthrography, ultrasound and MRI. Conventional MRI with the advent of surface coils have become valuable modality for good visualization of rotator cuff muscles and individual central tendon of four rotator cuff muscle.(3) However, its use as initial evaluation is limited because of it being expensive and not readily available.

Ultrasound is relatively noninvasive, inexpensive modality that can be used as first line modality. Initial sonographic results in the detection of rotator cuff tears varied.(4) probably due to the use of low frequency and low resolution $5 \mathrm{MHz}$ transducers and limited experience with the examination procedure. Subsequently, technical improvements such as $7.5-14 \mathrm{MHz}$ linear array broadbandwidth transducers and better penetration of the ultrasound beam as well as increased experience and detailed knowledge of shoulder anatomy and pathology significantly improved sonographic results and reliability.

Our study results comparing USG and MRI in rotator cuff pathologies showed equivocal distribution of rotator cuff pathologies and most of them are found above age of 60 years, correlating with studies by Ozaki et al.(5) and Needle et al.(6) the results of which proves that rotator cuff pathology does not show any gender differentiation. Factors for rotator cuff pathology like diminished vascularity in the cuff tissue and the extrinsic factors like impingement secondary to spurs, enthesophytes, downsloping acromion, type II and III of acromion increases with age.

In our study, the most commonly affected tendon is the supraspinatus followed by subscapularis and infraspinatus. Teres minor was not involved in any of the patients in this study. All the subscapularis and infraspinatus pathology has associated supraspinatus involvement. This is comparable to study by Zlatkin et al., where in they found supraspinatus tendon involvement in all rotator cuff pathologies.(7) and study by DePalma et al.(8) which showed supraspinatus as the commonly affected tendon.

Sensitivity, specificity and accuracy for diagnosis of full thickness tear of supraspinatus using USG compared to MRI were $100 \%$ showing that ultrasound is as good as MRI in diagnosing full thickness tear, whereas sensitivity, specificity and accuracy of diagnosing partial thickness tear using ultrasound compared to MRI are $100 \%, 78 \%$ and $84 \%$. There is no significant $\mathrm{p}$ value difference on comparing ultrasound with MRI on detecting tears. This is comparable to results of study by Rutten et al.(9) which showed sensitivity specificity and accuracy for full thickness tear using ultrasound were 95\%, 93\% and 94\%, whereas sensitivity, specificity and accuracy for partial thickness tear were $89 \%, 80 \%$ and $81 \%$. There was comparable diagnostic accuracy for detection of full thickness and partial thickness tear in their study except that the sensitivity for diagnosing partial thickness tear on USG is slightly better compared to MRI (67\% sensitivity), which was confirmed on surgery.

The 5-Ultrasound false positive diagnosis of partial thickness tear in our study cannot be confirmed as there is no surgical or arthroscopic correlation for these patients. Hence it is possible that ultrasound can be better than MRI in picking up intratendinous partial thickness tear and it can be used as additional modality to rule out tear in patients diagnosed as having tendinopathic changes alone in MRI.

A considerable number of supraspinatus tear patients (11 in USG and 7 in MRI) had associated tendinopathic changes. This correlated with a study by Neer.(10) who proposed shoulder impingement as a degenerative process starting as tendinopathy and progressing through partial thickness tear to full thickness tear.

Error in tear width measurement in ultrasound compared to MRI in our study is noted in 3 of 18 full thickness tears and 7 of 14 partial thickness tear showing there is a $50 \%$ error in assessment of partial thickness tear size, which can be due to irregular shape of cuff tear and irregular hypoechoic changes due to tendinopathic changes resembling tear. Tear size measurement errors in full thickness tear occurred in massive or large tear along curved surface and presence of significant associated bursal thickening.

It is important to assess tendon retraction of the medial edge of torn tendon as it is crucial for the surgical management. Sensitivity and specificity for detection of retracted tendon using ultrasound were 84 and 95\%. This correlated with a study by Teffey et al.(11) of 171 patients, on which ultrasound correctly predicted the retraction of $73 \%$ of full thickness tear patients. Thus the retracted tendon associated with rotator cuff tear can be effectively assessed by ultrasound without any significant $\mathrm{p}$ value difference as compared to MRI.

Examination of the muscles during shoulder sonographic examination was included in our study because of the potential clinical implications of muscle atrophy on the outcome of rotator cuff surgery. Sensitivity, specificity and accuracy for identifying muscle atrophy using ultrasound is $40 \%, 100 \%, 92 \%, 50 \%, 100 \%$ and $97 \%$ respectively. Although the sensitivity is less in ultrsound compared to MRI, there is no significant $\mathrm{p}$ value difference $(.250$ for supraspinatus and 1.000 for infraspinatus) indicating that ultrasound can also detect muscle atrophy.

Most of rotator cuff pathology in our study had associated acromioclavicular joint arthropathic changes. This correlated with a study by Needel et al.(12) In his study more than $80 \%$ patients with partial tear and all the patient with full thickness tear had acromioclavicular joint arthrosis. Sensitivity, specificity and accuracy for detecing AC joint changes using ultrasound in our study is $90 \%, 93 \%$ and $91 \%$ respectively.

Acromion are classified as type I to IV, flat, curved inferior surface, hooked and convex near the distal end respectively according to Bigliani and colleagues.(13) Variation in acromion configuration and slope with presence of enthesophytes drastically affects the space available for supraspinatus tendon, especially during abduction and rotational movements.(14) In our study of patients with supraspinatus pathology, type of acromion could not be assessed with ultrasound, however using the MRI 21 patients were identified as having type II, 7 as having type III, 2 as having os acromiale and 5 having type I and IV, which correlated with the above mentioned studies which showed type II and III acromions were most commonly associated with rotator cuff pathologies. 
The presence of enthesophytes with supraspinatus pathology were found in some patients using ultrasound, which showed sensitivity, specificity and accuracy of 35\%, $100 \%$ and $74 \%$ respectively compared to MRI and significant $\mathrm{p}$ value difference of .004 showing that MRI is superior to ultrasound in detecting enthesophytic changes of acromion.

In a study by Hollister et al.(15) done on 97 patients with surgically proven rotator cuff tear, $52 \%$ had fluid in the joint, bursa or both. It was concluded in this study that fluid in the bursa (Subacromial/subdeltoid) and joint effusion had strong association with rotator cuff tears, careful evaluation of cuff tendons is warranted to rule out tears in presence of joint effusion or bursal effusion. In our study sensitivity, specificity and accuracy for diagnosis of subacromial subdeltoid bursa, subcoracoid bursa and bicepital groove effusions were all above $90 \%$, whereas sensitivity, specificity and accuracy of detecting glenohumeral joint effusion were 50\%, 86\% and $82 \%$ respectively with no significant $\mathrm{p}$ value difference. This correlated with above mentioned studies which showed bursal changes and joint effusion were commonly associated with rotator cuff pathologies and diagnostic accuracy of ultrasound in assessing these are as good as MRI.

Coracoacromial ligament thickening is associated with impingement of supraspinatus tendon leading to supraspinatus pathologies and coracohumeral ligament thickening is associated with impingement of subscapularis pathology. Sensitivity, specificity and accuracy for identifying CAL thickness using USG in our study is $50 \%, 92 \%$ and $80 \%$ respectively; however, there is no significant $\mathrm{p}$ value difference when compared with MRI.

Subacromial impingement syndrome is a clinical entity that was proposed by Neer.(16) This syndrome is the result of chronic irritation of the supraspinatus tendon against the undersurface of the anterior one-third of the acromion, the coracoacromial ligament and the acromioclavicular joint. MRI is a reliable technique for the evaluation of the rotator cuff tendons, but it provides only a static evaluation of the shoulder joint and can only indirectly suggest the diagnosis of subacromial impingement because most findings are nonspecific. In 1990, Farin et al.(17) using dynamic sonography, described bursitis, fluid distention and pooling of fluid lateral to the subdeltoid bursa as signs of early-stage subacromial impingement, later wider range of abnormalities are identified which can indicate subacromial impingement. Dynamic sonography can provide direct visualization of the relationships between the anterior one-third of the acromion, subacromial bursa, supraspinatus tendon and greater tuberosity of the humeral head during active shoulder motion.

Diagnostic accuracy of dynamic evaluation using ultrasound in our patient for subcoracoid impingement is significantly high when compared to MRI with $p$ value difference of 0.004 indicating that ultrasound can be better modality in detecting subacromial impingement.

The coracohumeral distance assesses the space between the tip of the coracoid process and the lesser tuberosity and is ideally measured with the humerus in maximal internal rotation, for subcoracoid impingement. The average CHD is $11 \mathrm{~mm}$. Ultrasound and MRI showed all the patients with impingement equally with no significant $\mathrm{p}$ value difference. Ultrasound showed all the 4 patients (40\%) having reduced coracohumeral index causing encroachment of tendon, 3 patients (30\%) with pooling of fluid and severe pain, whereas MRI correctly identified reduced coracohumeral index in all the patients.

Our study has several important clinical implications. Firstly, a common approach towards the patients suspected of rotator cuff tears is to advise MRI (If available). Instead ultrasound could be advised which will save time, cost and improve clinical outcome of management. Secondly, patients with prosthesis, implants and claustrophobic patients which are the limitations of MRI can be benefited by ultrasound. The accuracy of ultrasound in experienced hands was found to be as good as that of MRI.(18) The MRI has shorter learning curve; it should be used secondarily and in selective cases because it provides more information about extent of tendons and has lower risk of artifacts. Due to the cost difference between the two procedures, our study clearly shows that ultrasound is more cost-effective test to use for identification of rotator cuff tears. It showed that ultrasound is accurate in diagnosing rotator cuff pathologies, which is in favorable comparison with study by Kenn et al. and Lach et al.(19,20) who proved ultrasound to be accurate and reliable in diagnosing a wide range of shoulder disorders compared with MRI.

There are several advantages of US over MRI. Ultrasound is available on a larger scale, portable, quick and a much more cost-effective imaging method, which is also easier tolerated by the patient. Ultrasound is not subject to motion artifacts, it allows instant comparison with the contralateral side, and tendons and other structures can be evaluated dynamically. The real time capability of US facilitates dynamic assesment for shoulder and allows better interaction with a patient who can point at the symptomatic area, which will optimize diagnostic yield. However, sonography of the shoulder joint is highly operator dependant. Small errors in transducer orientation and angulation may easily obscure small abnormalities within and around the cuff and give rise to false positive and false negative results.(21) But these potential pitfalls can be avoided by thoroughly understanding the normal anatomy, strictly insisting on proper transducer and patient position and using opposite shoulder for comparison. This can make sonography an effective, reliable and non-invasive means of detecting rotator cuff tears and as a first line tool in detecting rotator cuff tears than the expensive MRI and invasive artrography.

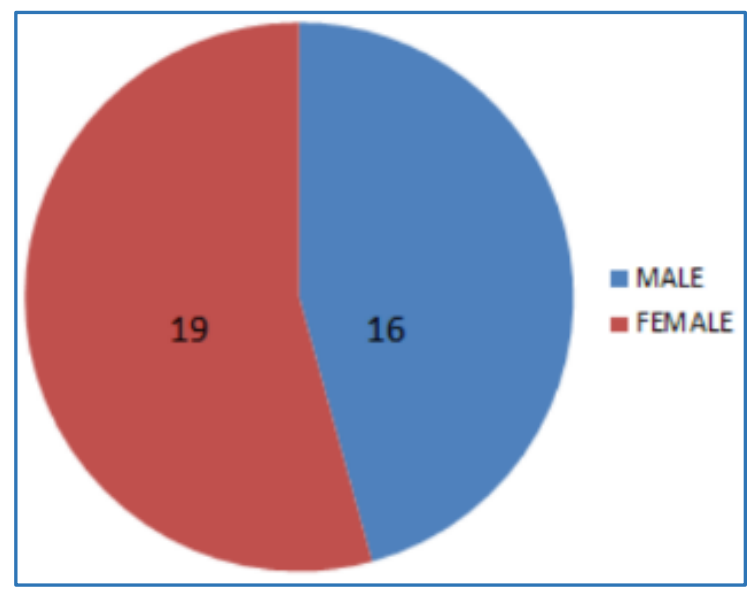

Gender Distribution of Rotator Cuff Pathologies 


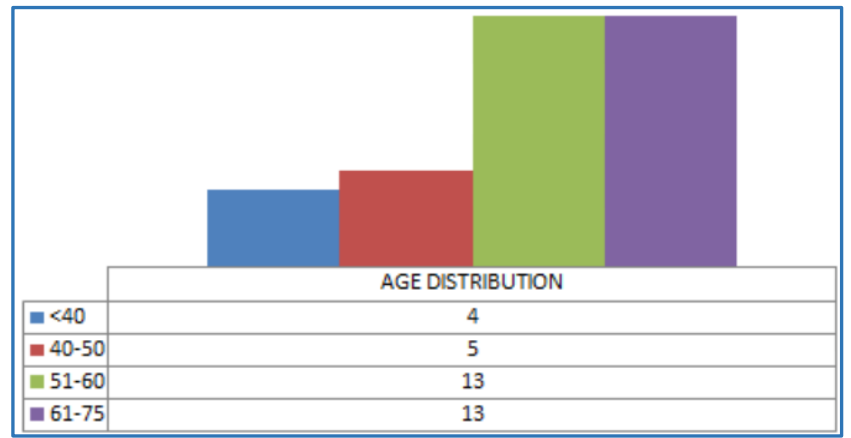

Agewise Distribution of Rotator Cuff Pathologies

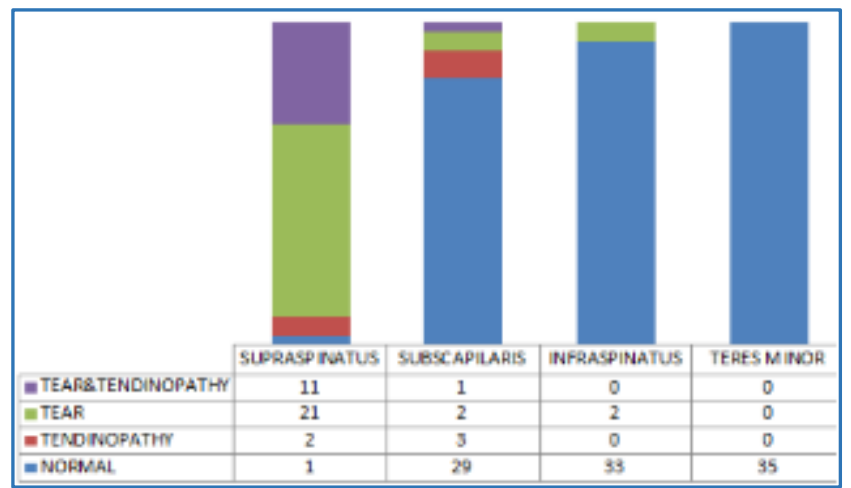

Distribution of Rotator Cuff Pathology in Ultrasound

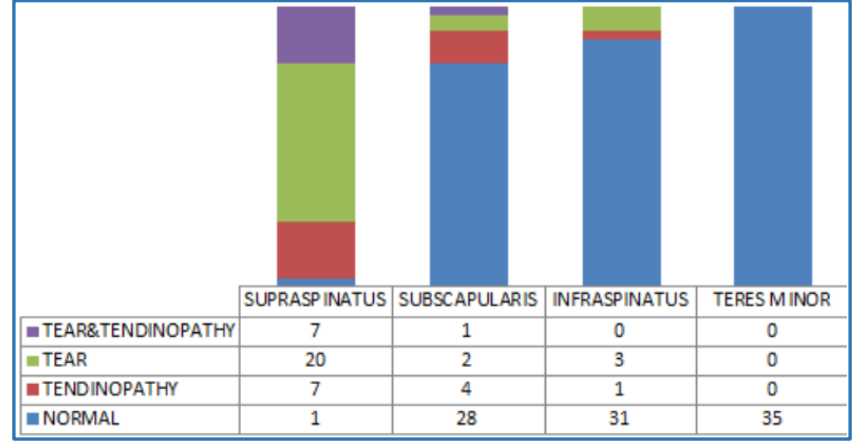

Distribution of Rotator Cuff Pathology In MRI

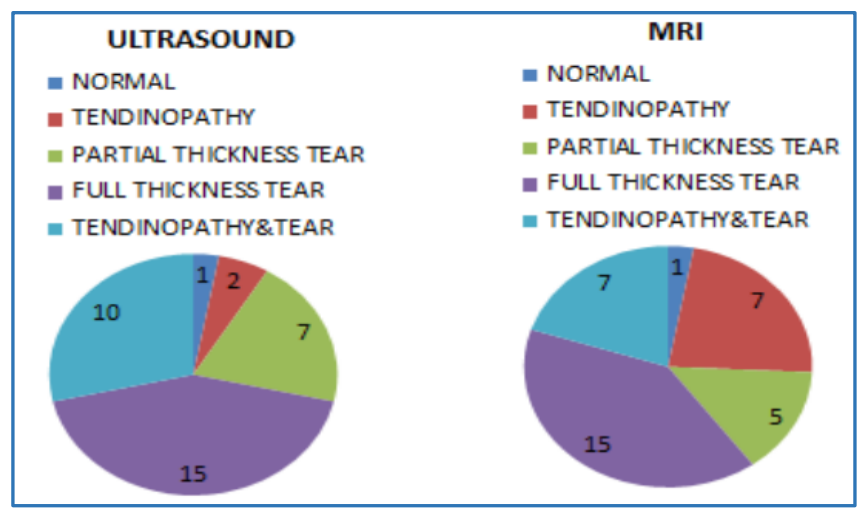

Supraspinatus Tendon pathology

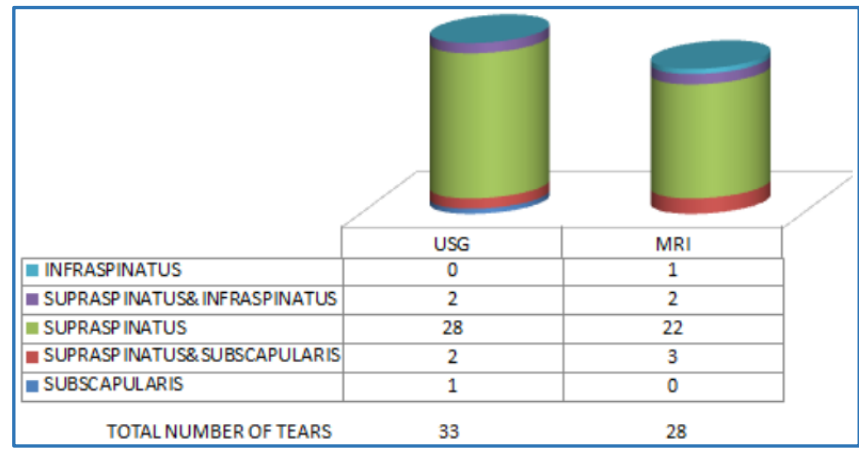

Distribution of Rotator Cuff Tears

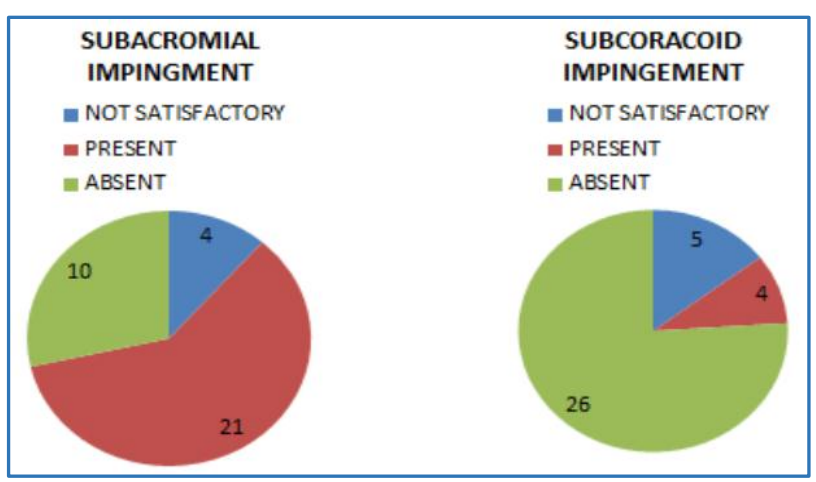

Dynamic Evaluation for Rotator Cuff Impingement Using Ultrasound

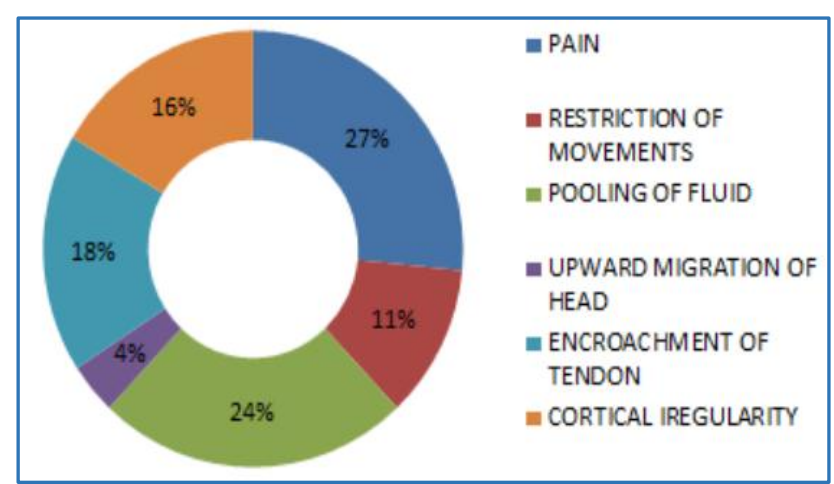

Distribution of Diagnostic Findings in Subacromial Impingement of Supraspinatus

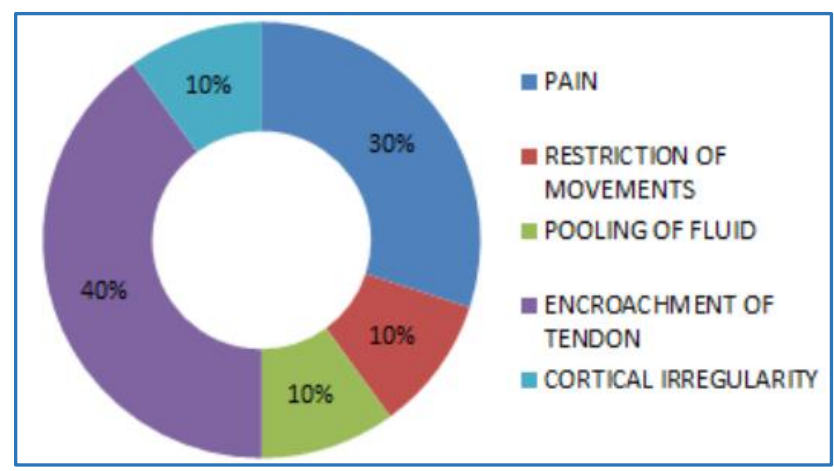

Distribution of Findings in Subcorachoid Impingement for Subscapularis 


\begin{tabular}{|c|c|c|c|c|c|c|c|c|}
\hline SUPRASPINATUS & TP & FP & TN & FN & SENSITIVITY\% & SPECIFICITY\% & ACCURACY \% & P Value \\
\hline $\begin{array}{c}\text { FULL THICKNESS } \\
\text { TEAR }\end{array}$ & 18 & 0 & 14 & 0 & 100 & 100 & 100 & 1.000 \\
\hline $\begin{array}{c}\text { PARTIAL THICKNESS } \\
\text { TEAR }\end{array}$ & 9 & 5 & 0 & 18 & 100 & 78 & 84 & 1.000 \\
\hline TENDINOPATHY & 12 & 1 & 20 & 2 & 86 & 95 & 91 & 1.000 \\
\hline $\begin{array}{c}\text { TENDON } \\
\text { RETRACTION }\end{array}$ & 11 & 1 & 21 & 2 & 84 & 85 & 91 & 1.000 \\
\hline MUSCLE ATROPHY & 2 & 0 & 30 & 3 & 40 & 100 & 91 & .250 \\
\hline $\begin{array}{c}\text { TEAR EXTENSION TO } \\
\text { SUBSCAPULARIS }\end{array}$ & 2 & 0 & 31 & 2 & 50 & 100 & 94 & .500 \\
\hline $\begin{array}{c}\text { TEAR EXTENSION TO } \\
\text { INFRASPINATUS }\end{array}$ & 2 & 0 & 33 & 0 & 100 & 100 & 100 & 1.000 \\
\hline
\end{tabular}

\begin{tabular}{|c|c|c|c|c|}
\hline & & MRI & & \\
\hline & & Positive & Negative & Total \\
\hline USG & Positive & 7 & 3 & 10 \\
\hline & Negative & 16 & 5 & 21 \\
\hline & Total & $\mathbf{2 3}$ & $\mathbf{8}$ & $\mathbf{3 1}$ \\
\hline \multicolumn{4}{|c|}{ Subacromial Impingement } \\
\hline
\end{tabular}

\begin{tabular}{|c|c|}
\hline & \\
\hline Sensitivity\% & $\mathbf{3 0}$ \\
\hline Specificity\% & 62 \\
\hline Accuracy\% & 38 \\
\hline P value & $\mathbf{. 0 0 4}$ \\
\hline \multicolumn{2}{|l}{} \\
\hline
\end{tabular}

\begin{tabular}{|c|c|c|c|c|}
\hline & \multicolumn{4}{|c|}{ MRI } \\
\hline & & Positive & Negative & Total \\
\hline USG & Positive & 2 & 2 & 4 \\
\hline & Negative & 1 & 27 & 28 \\
\hline & Total & $\mathbf{3}$ & $\mathbf{2 9}$ & $\mathbf{3 2}$ \\
\hline \multicolumn{5}{|c}{ Subcoracoid Impingement } \\
\hline
\end{tabular}

\begin{tabular}{|c|c|}
\hline & \\
\hline Sensitivity & $\mathbf{6 7}$ \\
\hline Specificity & 93 \\
\hline Accuracy & 90 \\
\hline P value & $\mathbf{1 . 0 0 0}$ \\
\hline \multicolumn{2}{|l}{} \\
\hline
\end{tabular}

\section{REFERENCES}

1. Resnick D. Shoulder arthrography. Radiol Clin North Am. 1981;19:243-52.

2. Middleton WD. Ultrasonography of the shoulder. Radiol Clin North Am 1992;30:927-40.

3. Roberts MC, Esterhai JC, Kressel HY, et al. High resolution surface coil MRI of the joints.

Radiographic 1983;176:37-38.

4. Brandt TD, Cardone BW, Grant TH, et al. Rotator cuff sonography: A reassessment.

Radiology 1989;173:323-327.

5. Rothmon RH, Parke W. The vascular anatomy of the rotator cuff. Clin Orthop 41:176-186, 1965.

6. McConville OR, Iannoni JP. Partial thickness tears of the rotator cuff: evaluation and management. J Am Acad Orthop Surgeons 7:32-43, 1999.

7. Depalma A, FJ. Surgery of the shoulder. Philadelphia, B Lippincott 1983;211-23.

8. Zlatkin MB. Rotator cuff tears diagnostic performance of MRI. Radiology:1989;172:223-229.

9. Rutten MJ, Spaargaren GJ, Van Loon T, de Waal Malefijt MC, Kiemeney LA, Jager GJ. Detection of rotator cuff tears: the value of MRI following ultrasound. Eur Radiol 2010 Feb;20(2):450-7.

10. Neer CS 2nd. Anterior acromioplasty for the chronic impingement syndrome in the shoulder: a preliminary report. J Bone Joint Surg Am 1972;54:41-50.

11. Teefey SA, Rubin DA, Middleton WD, Hildebolt CF, Leibold RA, Yamaguchi K. Detection and quantification of rotator cuff tears. Comparison of ultrasonographic, magnetic resonance imaging and arthroscopic findings in seventyone consecutive cases. J Bone Joint Surg Am 2004 Apr;86$\mathrm{A}(4): 708-16$

12. Steven DN, Micheal BZ, Jerry S, et al. MR Imaging of Rotator cuff: Peritendinous and Bone Abnormalities in Asymptomatic Population. AJR 1996;166:869-867.
13. Bigliani Lu, Morrison DS, April EW. The morphology of the acromion and its relationship to rotator cuff tears. Orthop Trans 1986;10:228.

14. Peterson CJ, Gentz CF. Ruptures of the supraspinatus tendon: the significance of distal acromioclavicular osteophytes. ClinOrthop 1983;174:143-148.

15. Mary SH, Laurence A, Randall MP, et al. Association of sonographically detected subacrommial subdeltoid bursal effusion and intraarticular fluid with rotator cuff tear. AJR 1995; Feb:165:605-608.

16. Neer CS 2nd. Anterior acromioplasty for the chronic impingement syndrome in the shoulder: a preliminary report. J Bone Joint Surg Am 1972;54:41-50.

17. Farin PU, Jaroma H, Harju A, et al. Shoulder impingement syndrome: sonographic evaluation.

Radiology 1990;176:845-849.

18. Zlatkin MB, Hoffman C, Shellock FG. Assessment of the rotator cuff and glenoid labrum using an extremity MR system: MR results compared to surgical findings from a multi-center study.

J MagnReson Imaging 2004;19:5:623-31.

19. Kenn W, Hufnagel P, Muller T, et al. Arthrography, ultrasound and MRI in rotator cuff lesions: a comparison of methods in partial lesions and small complete ruptures. Rofo 2000;172:3:260-6. 28.

20. Lach W, Dudarenko G, Brzosko M, et al. Usefulness of ultrasonographic and MR imaging in diagnosis of shoulder impingement. Chir Narzadow Ruchu Ortop Pol. 2003;68:5:307-11.

21. Middleton WD, Reinus WR, Totty WG, et al. Ultrasonographic evaluation of the rotator cuff and biceps tendon. J Bone Joint Surg [Am] 1986;68:440-450. 